\title{
BMJ Open Lubricant Investigation in Men to Inhibit Transmission of HPV Infection (LIMIT-HPV): design and methods for a randomised controlled trial
}

\author{
Cassandra Laurie, ${ }^{1}$ Mariam El-Zein (D) ${ }^{1}$ Joseph Tota, ${ }^{1}$ Pierre-Paul Tellier, ${ }^{2}$ \\ Francois Coutlée, ${ }^{3}$ Eduardo L Franco (D) , , Alexandra de Pokomandy (D) , ${ }^{2,4}$ LIMIT- \\ HPV study group
}

To cite: Laurie C, El-Zein M, Tota J, et al. Lubricant Investigation in Men to Inhibit Transmission of HPV Infection (LIMIT-HPV): design and methods for a randomised controlled trial. BMJ Open 2020;10:e035113. doi:10.1136/ bmjopen-2019-035113

- Prepublication history and additional material for this paper are available online. To view these files, please visit the journal online (http://dx.doi. org/10.1136/bmjopen-2019035113).

Received 18 0ctober 2019 Revised 18 February 2020 Accepted 27 February 2020

A Check for updates

(c) Author(s) (or their employer(s)) 2020. Re-use permitted under CC BY-NC. No commercial re-use. See rights and permissions. Published by BMJ.

For numbered affiliations see end of article.

\section{Correspondence to}

Dr Alexandra de Pokomandy; alexandra.depokomandy@ mcgill.ca

\section{ABSTRACT}

Introduction Gay, bisexual and other men who have sex with men (gbMSM) have an increased risk of human papillomavirus (HPV) infection and HPV-associated diseases, such as anal cancer and anogenital warts. A carrageenan-based lubricant could prevent HPV infection, thereby reducing the disease burden in this population. This paper describes the protocol for the Lubricant Investigation in Men to Inhibit Transmission of HPV Infection (LIMIT-HPV) study, an ongoing randomised controlled trial (RCT), evaluating efficacy of a carrageenan-based personal lubricant in reducing type-specific anal HPV incidence and prevalence among sexually active gbMSM, efficacy by HIV status, safety and tolerability of the gel and participant adherence to the intervention.

Methods and analysis The study is a double-blinded, placebo-controlled RCT. Volunteer gbMSM 18 years and older are randomly assigned 1:1 to receive the treatment (a self-applied anal microbicide gel with carrageenan) or placebo (a self-applied placebo gel). At each visit, computerised questionnaires are used to collect data on sociodemographic and clinical variables, lifestyle, sexual behaviour and the gels' safety and tolerability. At baseline and each follow-up visit (months 1, 2, 3, 6, 9 and 12), nurses collect anal specimens tested for 36 HPV types (linear array assay). HIV status is determined at baseline and 12 months. The primary outcome is incidence of type-specific anal HPV infection(s) undetected at baseline. Secondary outcomes are prevalence of type-specific anal HPV infection, safety, tolerability and adherence. We aim to recruit 380 participants to attain the study's objectives. Data will be analysed using intention-to-treat and perprotocol approaches with subgroup analyses by HIV status.

Ethics and dissemination Ethics approval was obtained by the Research Ethics Boards of McGill University, the McGill University Health Centre, Concordia University and Centre Hospitalier de I'Université de Montréal. Trial results will be disseminated through peer-reviewed publications and conference presentations.

Trial registration number NCT02354144.

\section{Strengths and limitations of this study}

- First study to explore the efficacy of carrageenan as a topical microbicide for preventing anal human papillomavirus (HPV) acquisition in gay, bisexual and other men who have sex with men (gbMSM).

- Randomised controlled trial design comparing carrageenan lubricant gel to placebo is optimal to evaluate the efficacy of carrageenan in gbMSM with and without HIV.

- Due to design limitations, dosage efficacy will not be evaluated.

The exact time of HPV acquisition will be unknown.

- The proportion of incident infections that could be due to reactivation of previously acquired HPV types is unknown.

\section{INTRODUCTION}

\section{Background and rationale}

Human papillomavirus (HPV) is one of the most common sexually transmitted infections (STIs) worldwide. ${ }^{1}$ A 2012 meta-analysis found that $93 \%$ of HIV-positive gay, bisexual and other men who have sex with men (gbMSM) and $65 \%$ of HIV-negative gbMSM are currently infected with HPV. ${ }^{2}$ Recently, an updated meta-analysis reported an HPV prevalence for HIV-positive and negative gbMSM of $81 \%$ and $47 \%$, respectively. ${ }^{3}$ Canadian statistics included in this meta-analysis were from a cohort study of HIV-positive gbMSM in Montreal, Quebec, which reported an HPV prevalence of $97.9 \%^{4}$ and a cross-sectional study in Vancouver, British Columbia, which reported an HPV prevalence of $78.6 \%$ and $56.9 \%$ among HIV-positive and negative gbMSM, respectively. ${ }^{5}$ There is overwhelming evidence that persistent HPV infection with high oncogenic risk HPV types is the primary risk factor leading to precancerous anal lesions. $^{6-15}$ 
While the incidence rate of anal cancer is 1-2 per 100000 per year, ${ }^{16}$ the rate is 5.1 per 100000 among HIV-negative gbMSM, and 45.9 per 100000 among HIVpositive gbMSM, based on multinational data. ${ }^{2}$ There is a lack of consensus on an anal screening strategy, and screening for high-grade lesions has not yet been shown to reduce the incidence of anal cancer. ${ }^{17}$ The risk of other HPV-related lesions, such as genital warts, may decrease with condom use, but there is no consensus on whether condom use decreases the risk of HPV positivity. ${ }^{18}$ Additionally, of the three current prophylactic HPV vaccines available, two are recommended for gbMSM $^{19}$ and offer protection from two (Gardasil) ${ }^{20}$ or seven (Gardasil 9) high-risk HPV types. ${ }^{21}$ There is thus a need for additional primary prevention measures.

Carrageenan, a gelling agent derived from red algae, is used as a stabiliser and emulsifier in food and cosmetic products. ${ }^{22}$ Previous research demonstrated that carrageenan can block HPV transmission in vitro ${ }^{23}$ and in animal studies. ${ }^{2425}$ Carrageenan interferes with virion surface proteins required for infection primarily by binding to the viral capsid thereby preventing attachment to the heparan sulphate proteoglycan receptor. ${ }^{23}$ This interaction is long enough to allow natural inactivation of HPV by the immune system, which may increase natural HPV clearance. ${ }^{26}$ The safety and acceptability of a carrageenan-containing gel was demonstrated for vaginal $^{27}$ and vaginal and penile use. ${ }^{28}{ }^{29}$ Because of the high prevalence of HPV and the greater risk of anal cancer and its precursor lesions in gbMSM, compared with men in the general population, it is critical to determine whether a carrageenan-based lubricant can prevent HPV transmission among this at-risk group. Moreover, as carrageenan's primary mechanism of action against HPV may be affected by innate and adaptive immunity, ${ }^{26}$ it is essential to verify if similar efficacy is observed in men with and without HIV. The aim of this paper is to describe the protocol for the 'Lubricant Investigation in Men to Inhibit Transmission of HPV Infection' (LIMIT-HPV) study, an ongoing, phase IIB, placebo-controlled, doubleblinded randomised controlled trial (RCT) to evaluate the effect of a carrageenan-based lubricant on anal HPV infections in gbMSM.

\section{Study objectives}

The primary objective is to evaluate the efficacy of carrageenan in reducing type-specific anal HPV incidence, that is, in preventing incident infections by HPV types undetected at baseline in sexually active gbMSM, overall and by HIV status. Secondary objectives are to: (1) evaluate the efficacy of carrageenan in reducing type-specific anal HPV prevalence, that is, in accelerating clearance of existing infections in sexually active gbMSM; (2) assess the safety and tolerability of the proposed gel; and (3) assess participant adherence to the intervention.

\section{METHODS AND ANALYSIS}

\section{Study design}

LIMIT-HPV is an exploratory, phase IIB, parallel group, block-randomised, placebo-controlled, RCT with 1:1 random assignment to the treatment (a self-applied anal microbicide gel with carrageenan) or placebo (a selfapplied placebo gel) group. The trial was registered on clinicaltrials.gov as of February 2015. Health Canada authorised the gel for use in a clinical trial (file number 169160).

\section{Patient and public involvement statement}

Prior to study initiation, a focus group was conducted to gather recommendations from 20 volunteer gbMSM and adapt our protocol accordingly. Participants answered a self-administered questionnaire, providing their perspective on sexual behaviour; lubricant and condom usage; candidate gels; partner's support and potential impact on compliance; sample collection; willingness to enrol in the trial; as well as other concerns and suggestions. This preliminary research in itself did not inform the research question; however, the trial design was directly impacted; for example, participants were asked about the maximum frequency they would be willing to have an anal specimen collected, which directly informed the frequency of testing in the actual RCT. Additionally, the question of whether the sample should be nurse collected rather than self-collected was supported by $6 / 20$ gbMSM, while 10/20 had no preference. Gel packaging was also adapted for their preferences. The recommended average monetary compensation to participate in the trial was $\$ 26.50$ per visit.

\section{Setting and recruitment}

Participants are recruited at the participating clinical sites or via advertisements in various media (classified ads on Kijiji, Craigslist and Les Pacs; Facebook; Fugues magazine, Quebec's gay and lesbian magazine; McGill and Concordia Classifieds; an interview on McGill/Montreal CKUT Campus Community radio station; promotional videos; 'What's New' blurbs emailed to McGill students; study announcements emailed to Université de Montréal students; and class presentations) and through printed promotional materials, including posters, business cards, posters and button pins. Study recruitment began in February 2016 and study visits are conducted at the following clinical sites: McGill University Health Centre (MUHC), Clinique Médicale Urbaine du Quartier-Latin, Clinique OPUS, McGill Health Service Clinic, Concordia Health Services or at the Gerald Bronfman Department of Oncology at the Division of Cancer Epidemiology of McGill University.

\section{Study population and procedures}

Individuals are screened directly for eligibility at the clinical sites or prior to that over the telephone (online supplementary appendix 1). Alternatively, subjects interested in the study can first fill out an online, self-administered 


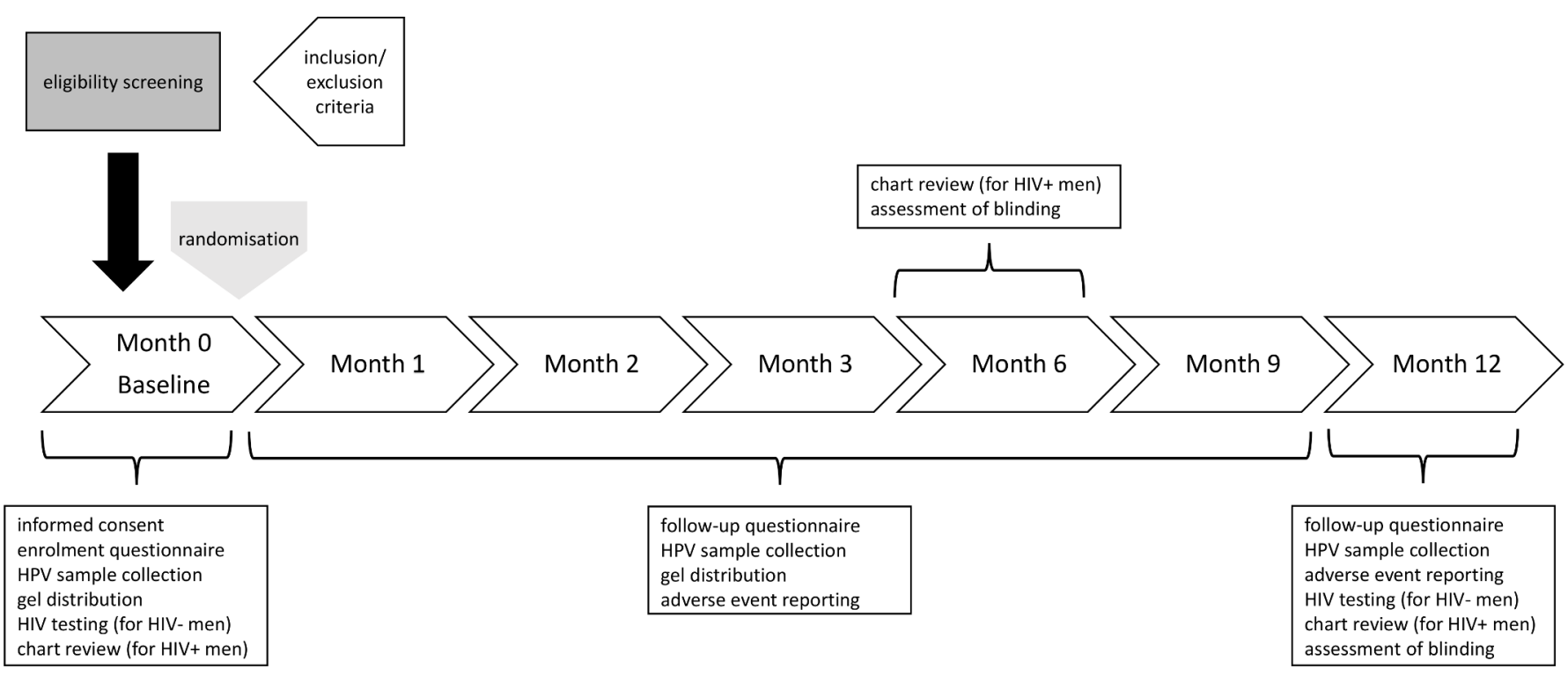

Figure 1 Potential participants are screened for eligibility based on predefined inclusion and exclusion criteria. Eligible participants provide informed consent and are randomised 1:1 to receive either the carrageenan-based gel or the placebo gel. They fill out an enrolment questionnaire, provide an anal HPV sample, receive a supply of the gel, are tested for HIV if HIVnegative and a chart review is completed if HIV-positive. The participants return for each follow-up visit (months 1, 2, 3, 6, 9 and 12). At follow-up visits (months 1-9), participants fill out a follow-up questionnaire, provide an HPV sample, are provided gel and report on adverse events. At month 6 and 12, there is a blinding assessment and a chart review is completed for HIV-positive participants. At month 12, participants fill out a follow-up questionnaire, provide an HPV sample and report on adverse events, and HIV-negative men are tested again for HIV.

eligibility pre-enrolment questionnaire (online supplementary appendix 2). If eligible, they are contacted to confirm their eligibility and schedule the enrolment visit. Otherwise, they are emailed to thank them for their interest and explain their ineligibility.

Eligibility is based on the following criteria:

- Men aged 18 or older.

- Living in Montreal and planning to remain in the city for the next 12 months.

- Having had receptive anal sex with one or more men during the previous 3 months and intend to continue being sexually active for the duration of their involvement in the study, irrespective of whether their sexual partner will change.

- Planning on having receptive anal sex with one or more men but less than 50 different partners per year.

- Understanding French or English.

- Willing to follow study instructions and comply with follow-ups for 12 months.

- Willing to do an HIV test (for men who were never tested seropositive for HIV).

Exclusion criteria:

- Participants must not be receiving treatment for anal or perianal condylomas or anal intraepithelial neoplasia lesions during the trial.

- Must not have a known allergy or hypersensitivity to any of the ingredients in either gels.

Study procedures according to each visit are summarised in figure 1. Eligible men attend an enrolment visit, where the research nurse obtains written, site-specific informed consent (online supplementary appendix 3
McGill site) and instructs the participant on proper gel use. A 1-month gel supply is provided, and the first specimen is collected. The nurse also provides details about HPV infection and advice about condom use and sexual health (ie, importance of condom use to prevent HIV and other STIs). At subsequent visits, additional bottles of gel are provided, and patients are reminded to use the gel.

\section{Randomisation and blinding}

Once written informed consent is obtained and HIV status is confirmed, participants are randomised 1:1 to receive either a carrageenan-containing gel or a placebo gel. Intervention assignment occurs via a computerassisted block randomisation with randomly variable block sizes. Each participant is assigned an individual code for the duration of the study, which is used to match him to the study arm. The trial is double blinded: participants, care providers, investigators and outcomes assessors are unaware of treatment allocation. To ensure blinding, the two gels and their containers look and feel almost identical. Additionally, four random product codes are assigned to the treatment gel and a different set to the control gel (eight in total) to minimise the risk of unblinding. The success of blinding is evaluated at 6 and 12 months by asking subjects to guess their assignment. If the majority guess correctly, it would suggest that blinding was ineffective.

\section{Intervention}

The intervention and placebo gels used in this trial are two commercially available gels. The differentiating feature 
is that one gel contains carrageenan (intervention) and the other does not (placebo). Both gels are water based, latex condom compatible, clear, odourless, tasteless and have similar viscosity. Both are packaged in a plastic bottle with a disk cap that can be operated with one finger and must be applied prior to receptive anal intercourse (RAI) during the entire study period. Participants are instructed to dispense around $15 \mathrm{~mL}$ of the gel into the hand and apply directly to genital, anal and condom surfaces prior to and as needed during RAI. When sexual activity ceases, the water-based formulation of the gel allows it to be easily removed with lukewarm water. Participants are asked to use the assigned gel for the entire 12 months of follow-up, independently of other methods of protection against STIs (eg, condoms).

\section{Adherence}

To improve adherence, participants are provided with an unlimited gel supply until the end of the study. Up until April 2019, a monetary compensation of $\$ 25 /$ visit was provided to each participant. This amount was since increased to $\$ 50$ for visits 1 and 7 and $\$ 40$ for visits $2-6$ to better reflect the market for compensation in clinical research, to improve recruitment and to help retain participants.

\section{Concomitant care}

The nurse informs unvaccinated individuals that the HPV vaccine has now been approved for men between 9 and 26 years of age and reminds them that protection is prophylactic and restricted to nine vaccine-target types. In addition to the required intervention gel, we recommend condom use for the prevention of HIV and other STIs. Condoms are easily accessible: many community organisations in Montreal such as REZO, a communitybased organisation dedicated to health promotion and prevention of HIV/AIDS and other STIs, already provide condoms free of charge as a public health intervention. We also offer participants with latex allergies non-latex condoms free of charge that are compatible with the study gels. Condoms are available from the study nurse on request.

\section{Sample size}

Data from the Montreal (Human Immunodeficiency and Papilloma Virus Research Group) HIPVIRG cohort study of gbMSM living with $\mathrm{HIV}^{4}$ and a multinational metaanalysis representing both gbMSM subgroups ${ }^{2}$ informed our calculation of sample size. The reported prevalence in the HIPVIRG population ${ }^{4}$ was very similar to studies that were conducted outside of Montreal in gbMSM living with HIV, ${ }^{2}$ justifying adopting incidence data from gbMSM without HIV from settings outside of Montreal. The technique of Dupont and Plummer ${ }^{30}$ was used to estimate the hazard rate of acquisition. Among HIV-negative gbMSM, we estimated a conservative preventive effect size of $50 \%$ based on the expert opinion of Dr John Schiller, who discovered carrageenan's inhibitory properties (personal communication). ${ }^{23}$ We expect a lower effect size of $30 \%$ among HIV-positive gbMSM, as carrageenan's primary inhibition mechanism relies on the immune response. The power calculations were separately tailored to satisfy our primary endpoint in each gbMSM population; however, if results are homogeneous across groups, we will consider pooling results to improve the precision of our estimates. Additionally, we specified $80 \%$ power to evaluate our primary objective with a type 1 error of 0.05 and two-sided hypothesis. Assuming an incidence proportion of $30 \%$ at 12 months among HIV-negative gbMSM $^{2}$ and accounting for $10 \%$ loss to follow-up, the sample size required for an effect size of $50 \%$ was calculated to be 270 . Similarly, assuming an $85 \%$ incidence of HPV infection at 12 months among HIV-positive gbMSM ${ }^{2}$ and accounting for $10 \%$ loss to follow-up, the estimated sample size required for an effect size of $30 \%$ was calculated to be 107 . Hence, recruiting 380 participants (110 HIV-positive and 270 HIV-negative) would ensure sufficient power at the end of follow-up to assess the study's objectives. With the high frequency of new sex partners among gbMSM in a similar study by our group, ${ }^{4}$ a 1 -year follow-up period would be sufficient to allow HPV exposure opportunity and evaluate compliance.

\section{Data collection}

The initial visit takes approximately $30 \mathrm{~min}$, while all subsequent follow-up visits (1, 2, 3, 6, 9 and 12 months) require about $20 \mathrm{~min}$ each. Men are asked to abstain from RAI and gel use 48 hours before specimen collection to minimise the risk of contamination. ${ }^{31}$

\section{Computerised questionnaire}

Participants complete a self-administered baseline questionnaire at enrolment and six follow-up questionnaires (online supplementary appendices 4 and 5 , respectively). These measure HPV risk factors, compliance and monitor the gels' safety and tolerability. Between follow-up visits, participants are asked to $\log$ into a secure web module at least once a week to answer questions on daily sexual activities, condom and study gel use and adverse events (AEs). To minimise recall bias, information can only be updated for the past 7 days (incomplete surveys expire after a week). Web-based diaries have been shown to be effective for logging sexual activities and superior to questionnaires completed during visits for reducing recall bias. ${ }^{32}$ This ensures high compliance and improves data quality. Responses are employed to evaluate adherence and assist in developing future studies.

\section{Reporting AEs}

To gauge the severity of AEs related to the study intervention, we refer to the Rectal Genital Grading Table for Use in Microbicide Studies ${ }^{33}$ and Male Genital Grading Table for Use in Microbicide Studies. ${ }^{34}$ If a stable, chronic condition is noted in the enrolment medical history questionnaire but does not exacerbate during the trial, symptoms are recorded in the $\mathrm{AE}$ report but are not considered to 
be attributable to the gel. Subjects are advised to promptly notify the nurse of any AE; the event is documented, and the participant is triaged and treated at the discretion of the study physicians. Nonetheless, should subjects fail to immediately report an $\mathrm{AE}$, they are also asked about any recent medical visits/AEs at each follow-up visit in the questionnaire.

\section{Anal sample collection}

HPV infection status is assessed by testing anal specimens. Trained study nurses collect specimens according to the Protocol for Anal Swab Collection (online supplementary appendix 6). ${ }^{4}$ The swab sample is immediately preserved in PreservCyt and kept at $4^{\circ} \mathrm{C}$ pending transfer to FC's laboratory, a WHO-accredited HPV diagnostics centre. Samples are batched and transported every 2-3 months.

\section{HPV DNA detection and typing}

The swab sample is subject to centrifugation at $13000 \mathrm{~g}$ for $15 \mathrm{~min}$ at $22^{\circ} \mathrm{C}$; the supernatant is discarded, and the pellet is resuspended in $300 \mu \mathrm{L}$ of $20 \mathrm{mmol} / \mathrm{L}$ Tris buffer ( $\mathrm{pH}$ 8.3). DNA is purified using a Master-Pure Kit (Epicentre) and tested in each PCR assay. ${ }^{35}$ HPV detection and typing is done via the PGMY PCR protocol coupled with the linear array method, commercially available from Roche. ${ }^{36}$ This test permits testing and typing for 36 different genital HPV types. ${ }^{36}$ These types can be categorised into three alphapapillomavirus subgenera based on oncogenicity and tissue tropism: subgenus 1 includes low oncogenic risk types (HPVs 6, 11, 40, 42, 44 and 54), subgenus 2 includes high oncogenic risk types (HPVs 16, 18, 26, 31, 33, 34, 35, 39, 45, 51, 52, 53, 56, $58,59,66,67,68,69,70,73$ aand 82 ), and subgenus 3 includes mostly commensal types (HPVs $61,62,71,72$, $81,83,84$ and 89$).{ }^{37-40}$

\section{HIV testing}

For participants who report being HIV-negative, the nurse performs a rapid HIV test at baseline and at 12 months, as is standard of care in high-risk populations (online supplementary appendix 7 ). If positive, the participant is referred immediately to AdP at the MUHC to ensure rapid engagement with HIV care. For HIV-positive participants, a brief chart review is done at 0,6 and 12 months to collect information on CD4 count, HIV viral load and current antiretroviral regimen.

\section{Loss to follow-up}

Discontinuing participation of a study subject occurs if the participant voluntarily withdraws from the trial, or has AEs, illness or other medical conditions determined by a physician to be serious enough to terminate his involvement in the study. Loss to follow-up is described as failure to reach a participant for a follow-up visit 6 months postrandomisation, or the potential for a participant to jeopardise the study's integrity through protocol non-compliance.

\section{Outcome measures}

The primary outcome is presence of a newly detected anal infection of a specific HPV type(s) in an individual who was negative for that HPV type(s) at enrolment. The secondary outcome is clearance of type-specific anal HPV infections found at baseline. Analyses will be conducted for a conservative (one negative HPV result after a positive result) and liberal (two consecutive negative results after a positive result) definition of clearance. Other secondary outcomes include participant adherence and AEs reporting.

\section{Data management}

Study and data management are facilitated through the use of a secure, password-protected web-based database to record and manage study procedures. The database is used to record participant and clinic visit information, plan visits and export data. It is only accessible from specific IP addresses. A coded numeric system is used to identify subjects. All data, including but not limited to records, case report forms and laboratory results, remain confidential and stored in a secure location. Research staff are the only individuals with access to these personal documents. They are available to the study sponsor or participating regulatory agencies on request. For quality control, data are downloaded from the server each month and checked for possible errors. Data management is done using SAS V.9.4. Any missing data will be handled by multiple imputations if appropriate.

\section{Data analysis}

Analyses will be conducted separately among gbMSM with and without HIV and pooled if appropriate. These will use intention-to-treat (ie, including all participants who were randomised and received at least 1 month's supply of gel) and per-protocol (ie, including only 'adherent' participants who complied with the protocol) approaches. Because of randomisation, we expect the rates of type-specific HPV infections to be comparable between study arms at enrolment.

\section{Primary aim 1 (prevention)}

Carrageenan's efficacy will be evaluated by testing the null hypothesis of no difference in time to anal type-specific HPV incident infection between treatment groups using the log rank test. Time to HPV infection will be defined as the difference in days between an incident HPV detection date and time zero at enrolment. We will use Cox proportional hazards regression to estimate the HR and $95 \%$ CI of HPV infection for treatment versus placebo. If the proportionality assumption is not met or the HR changes over time, we will fit a discrete-time hazards model. ${ }^{41}$

A sensitivity analysis will be conducted restricting to the most adherent participants in terms of gel usage. Adherence will be calculated as the number of times the gel was used during RAI divided by the number of RAIs reported in the same interval. A participant will be considered adherent if he reported, as recommended, gel use at 
least $>50 \%$ of the time prior to every act of intercourse. Additional analyses will allow for time-varying adherence, defined as adherence since the last administered questionnaire.

\section{Secondary aim 1 (clearance)}

Time-to-event analysis techniques will be used to measure type-specific clearance of HPV infections present at enrolment, according to the intervention. Time to clearance and HRs of clearance will be calculated as above.

\section{Secondary aim 2 (safety, tolerability and adherence)}

Safety and tolerability of the interventions will be evaluated using the $\mathrm{AE}$ reports from both groups. For each participant, mean adherence will be calculated for the time period between two consecutive visits and for the whole follow-up period, and it will be compared between the intervention and placebo groups using a t-test. If adherence is not normally distributed, median adherence will be compared between groups using the MannWhitney test. As mentioned previously, adherence will also be evaluated as a binary variable and compared between groups using the $\chi^{2}$ test for each interval and overall.

\section{Monitoring}

An independent data safety monitoring board oversees the trial to ensure that it is conducted in accordance with the ethical principles of good clinical practice. The board will review the results of the interim analysis and make recommendations regarding safety concerns and/or suspension or early termination of the study (eg, unequivocal evidence of efficacy). The same board members also oversee the Carrageenan gel Against Transmission of Cervical HPV (CATCH) RCT, which is similar in design to LIMIT-HPV; however, it evaluates the efficacy of a carrageenan gel among heterosexually active women. ${ }^{42}$

\section{ETHICS AND DISSEMINATION}

This is the seventh study protocol version, last revised 30 January 2019. When $50 \%$ of the targeted population (380 gbMSM) are recruited, an interim analysis will be conducted. Reports of trial findings-in the form of abstracts and manuscripts to be submitted, respectively, to peer-reviewed journals and conferences-will be presented according to the CONsolidated Standards of Reporting Trials statement. ${ }^{43}$ The coinvestigators involved in the study will assist in dissemination of research findings directly to health clinics and the gbMSM community.

\section{DISCUSSION}

Presently, there is no effective way to treat anal HPV infections. With the potential for broad-spectrum anti-HPV activity, carrageenan could be a useful adjunct to HPV vaccination as a primary means of preventing HPV infections. Given the high burden of HPV infections in the gbMSM community, regular application of a carrageenanbased lubricant could be a cost-effective preventive approach, especially considering that most gbMSM regularly use lubricants for anal sex. Furthermore, treatments for condyloma and high-grade lesions are costly and often need to be repeated, as the recurrence rate is very high (particularly among people with HIV) ${ }^{44}$ Also, vaccination is generally only maximally effective at preventing infection if administered prior to becoming sexually active. ${ }^{45}$

To the best of our knowledge, the LIMIT-HPV study is the first to test carrageenan against anal HPV infections. Its main strength is the blinded RCT design. Additionally, considering HIV-positive and negative gbMSM would allow for the evaluation of the gel's efficacy in both groups. There are study limitations. An evaluation of dosage efficacy is not possible, as we do not collect information on the exact amount of gel used. While biannual ${ }^{46-48}$ and annual ${ }^{49-53}$ anal HPV sampling in longitudinal studies is common, that length of follow-up will not give sufficient detail to evaluate the study's objectives. In an ideal research setting, HPV status would be ascertained daily to have a more precise measurement of the time of HPV acquisition; however, to minimise burden on the patient, the current schedule was deemed optimal. HPV incidence is consequently interval censored, that is, infection date occurs sometime between the last negative and the first positive test, but the exact date is unknown. However, as the time interval between each visit is relatively short, the interval would represent an appropriate approximation. An additional limitation is the possibility that some "incident' HPV infections are due to reactivation of previously acquired HPV, as opposed to acquisition from sexual activity ${ }^{54}$ However, because the proportion of incident infections that could be due to viral latency is expected to be balanced between groups as a result of (successful) randomisation, the effect on the risk estimate could be biased towards the null.

The LIMIT-HPV study may show a similar protective effect as was demonstrated in an interim analysis of a related study (CATCH RCT) conducted by our team. A reduction in the risk of incident HPV infection among participants randomised to the carrageenan gel was demonstrated, and importantly, the gels appeared safe: none of the reported $\mathrm{AE}$ were attributed to the gels. ${ }^{42}$ If efficacy of the carrageenan gel is demonstrated, the current trial has the potential to improve the health of individuals in the gbMSM community by providing protection against all HPV genotypes and ultimately reducing the risk of HPV-associated diseases in this at-risk group.

\section{Author affiliations}

${ }^{1}$ Division of Cancer Epidemiology, McGill University, Montreal, Quebec, Canada ${ }^{2}$ Department of Family Medicine, McGill University, Montreal, Quebec, Canada ${ }^{3}$ Service de Microbiologie Médicale et Service d'Infectiologie, Départements de Médecine et de médecine de laboratoire, Centre Hospitalier de L'Universite de Montreal, Montreal, Quebec, Canada

${ }^{4}$ Research Institute of the McGill University Health Centre, Montreal, Quebec, Canada

Correction notice This article has been corrected since it was published. 6th author's name has been corrected. 
Acknowledgements We wish to thank the volunteering participants and the following employees of the Lubricant Investigation in Men to Inhibit Transmission of HPV Infection (LIMIT-HPV) study: Jennifer Selinger, Maude Pastor, Abbie Chan and Parker Tope for study promotion; Deisy Bustillo-Dominguez and Catherine Nguyen-Huy for temporary management of subject participation and specimen collection (filling in for a maternity leave). The authors would also like to thank Doris Edmond (Student Health Services Clinic, Concordia University) and the staff of the Student Health Services Clinics at McGill and Concordia universities for their collaboration.

Collaborators LIMIT-HPV study team members: Affiliated with the Division of Cancer Epidemiology, McGill University, Montréal, Canada: Allita Rodrigues (study coordinator); Natalia Morykon and Raphaela Rodrigues (management of subject participation and specimen collection); Sheila Bouten and Samantha Shapiro (data management). Affiliated with Clinique OPUS: Roger Leblanc; Affiliated with Clinique Médicale Urbaine du Quartier Latin: Benoit Trottier (clinical collaborators). Affiliated with the Research Institute of the McGill University Health Centre, Montréal, Québec, Canada: Christina de Castro and Karène Proulx-Boucher (study coordination and management of subject participation); Guillaume Theriault (specimen collection). Affiliated with the Service de Microbiologie Médicale et service d'Infectiologie, Départements de Médecine et de Biologie médicale, Centre Hospitalier de I'Université de Montréal, Montréal, Québec, Canada: Julie Guénoun (HPV testing and genotyping).

Contributors EF, AdP, FC and P-PT conceived and designed the study. JT contributed to the grant application writing. ME-Z managed the study. CL drafted the manuscript under the supervision of EF, AdP and ME-Z. All authors reviewed the manuscript and approved the final version.

Funding The LIMIT-HPV study was funded by the Canadian Institutes of Health Research (CIHR) (grant MOP-137066 to AdP and ELF, grant FDN-143347 to EF), the Canadian Cancer Society Research Institute (grant 703032 to EF) and an HIV/ HPV grant from the HIV/AIDS network of Fonds de Recherche du Quebec - Sante (FRQ-S; to AdP). CarraShield Labs Inc (St. Petersburg, Florida) provided gel supplies and non-latex condoms at no cost. CIHR and CarraShield Labs Inc were not involved in study design or data collection and will not be involved in data analysis or preparation of results. AdP holds a salary award (chercheur-boursier) from the FRQ-S.

Competing interests AdP's clinic participates in pharmaceutical clinical trials for HIV antiretrovirals and HCV treatments (ViiV Healthcare, Janssen, Merck and Gilead), received honoraria for consulting on HIV antiretroviral regimen for ViiV Healthcare, and received grants from CIHR and FRQ-S outside the submitted work. EF reports grants and personal fees from Merck, grants, personal fees and non-financial support from Roche and personal fees from GSK, outside the submitted work. $\mathrm{JT}$ is a Merck employee. FC reports grants from Réseau FRQS-SIDA during the conduct of the study and grants to his institution for HPV-related work but outside of the submitted work from Merk Sharp and Dome, Roche Diagnostics and Becton Dickinson.

Patient consent for publication Not required.

Provenance and peer review Not commissioned; externally peer reviewed.

Open access This is an open access article distributed in accordance with the Creative Commons Attribution Non Commercial (CC BY-NC 4.0) license, which permits others to distribute, remix, adapt, build upon this work non-commercially, and license their derivative works on different terms, provided the original work is properly cited, appropriate credit is given, any changes made indicated, and the use is non-commercial. See: http://creativecommons.org/licenses/by-nc/4.0/.

ORCID iDs

Mariam El-Zein http://orcid.org/0000-0002-5190-0370

Eduardo L Franco http://orcid.org/0000-0002-4409-8084

Alexandra de Pokomandy http://orcid.org/0000-0001-6809-1357

\section{REFERENCES}

1 de Sanjosé S, Diaz M, Castellsagué X, et al. Worldwide prevalence and genotype distribution of cervical human papillomavirus DNA in women with normal cytology: a meta-analysis. Lancet Infect Dis 2007;7:453-9.

2 Machalek DA, Poynten M, Jin F, et al. Anal human papillomavirus infection and associated neoplastic lesions in men who have sex with men: a systematic review and meta-analysis. Lancet Oncol 2012;13:487-500.
3 Marra E, Lin C, Clifford GM. Type-Specific anal human papillomavirus prevalence among men, according to sexual preference and HIV status: a systematic literature review and meta-analysis. $J$ Infect Dis 2019;219:590-8.

4 de Pokomandy A, Rouleau D, Ghattas G, et al. Prevalence, clearance, and incidence of anal human papillomavirus infection in HIV-infected men: the HIPVIRG cohort study. J Infect Dis 2009;199:965-73.

5 Gilbert M, Kwag M, Mei W, et al. Feasibility of incorporating selfcollected rectal swabs into a community venue-based survey to measure the prevalence of HPV infection in men who have sex with men. Sex Transm Dis 2011;38:964-9.

6 Caussy D, Goedert JJ, Palefsky J, et al. Interaction of human immunodeficiency and papilloma viruses: association with anal epithelial abnormality in homosexual men. Int $J$ Cancer 1990;46:214-9.

7 Chin-Hong PV, Vittinghoff E, Cranston RD, et al. Age-Related prevalence of anal cancer precursors in homosexual men: the explore study. J Natl Cancer Inst 2005;97:896-905.

8 Ferenczy A, Franco E. Persistent human papillomavirus infection and cervical neoplasia. Lancet Oncol 2002;3:11-16.

9 Friedman HB, Saah AJ, Sherman ME, et al. Human papillomavirus, anal squamous intraepithelial lesions, and human immunodeficiency virus in a cohort of gay men. J Infect Dis 1998;178:45-52.

10 Melbye M, Palefsky J, Gonzales J, et al. Immune status as a determinant of human papillomavirus detection and its association with anal epithelial abnormalities. Int J Cancer 1990;46:203-6.

11 Palefsky JM, Gonzales J, Greenblatt RM, et al. Anal intraepithelial neoplasia and anal papillomavirus infection among homosexual males with group IV HIV disease. JAMA 1990;263:2911-6.

12 Frazer IH, Medley G, Crapper RM, et al. Association between anorectal dysplasia, human papillomavirus, and human immunodeficiency virus infection in homosexual men. Lancet 1986;2:657-60.

13 Palefsky JM. Anal squamous intraepithelial lesions: relation to HIV and human papillomavirus infection. J Acquir Immune Defic Syndr 1999;21 Suppl 1:S42-8.

14 Palefsky JM, Holly EA, Ralston ML, et al. Anal cytological abnormalities and anal HPV infection in men with centers for disease control group IV HIV disease. Genitourin Med 1997;73:174-80.

15 Ryan DP, Compton CC, Mayer RJ. Carcinoma of the anal canal. N Engl J Med 2000;342:792-800.

16 Grulich AE, Poynten IM, Machalek DA, et al. The epidemiology of anal cancer. Sex Health 2012;9:504-8.

17 Pernot S, Boucheron P, Péré H, et al. Comparison of anal cancer screening strategies including standard anoscopy, anal cytology, and HPV genotyping in HIV-positive men who have sex with men. $\mathrm{Br} J$ Cancer 2018;119:381-6.

18 Manhart LE, Koutsky LA. Do condoms prevent genital HPV infection, external genital warts, or cervical neoplasia? A meta-analysis. Sex Transm Dis 2002;29:725-35.

19 Petrosky E, Bocchini JA, Hariri S, et al. Use of 9-valent human papillomavirus (HPV) vaccine: updated HPV vaccination recommendations of the Advisory Committee on immunization practices. MMWR Morb Mortal Wkly Rep 2015;64:300-4.

20 Giuliano AR, Palefsky JM, Goldstone S, et al. Efficacy of quadrivalent HPV vaccine against HPV infection and disease in males. $N$ Engl $J$ Med 2011;364:401-11.

21 Joura EA, Giuliano AR, Iversen O-E, et al. A 9-valent HPV vaccine against infection and intraepithelial neoplasia in women. $N$ Engl J Med 2015;372:446-8.

22 Coggins C, Blanchard K, Alvarez F, et al. Preliminary safety and acceptability of a carrageenan gel for possible use as a vaginal microbicide. Sex Transm Infect 2000;76:480-3.

23 Buck CB, Thompson CD, Roberts JN, et al. Carrageenan is a potent inhibitor of papillomavirus infection. PLoS Pathog 2006;2:e69.

24 Roberts JN, Buck CB, Thompson CD, et al. Genital transmission of $\mathrm{HPV}$ in a mouse model is potentiated by nonoxynol-9 and inhibited by carrageenan. Nat Med 2007;13:857-61.

25 Roberts JN, Kines RC, Katki HA, et al. Effect of Pap smear collection and carrageenan on cervicovaginal human papillomavirus-16 infection in a rhesus macaque model. J Natl Cancer Inst 2011;103:737-43.

26 Tobacman JK. The common food additive carrageenan and the alpha-Gal epitope. J Allergy Clin Immunol 2015;136:1708-9.

27 Kilmarx $\mathrm{PH}$, van de Wijgert JHHM, Chaikummao S, et al. Safety and acceptability of the candidate microbicide Carraguard in Thai women. J Acquir Immune Defic Syndr 2006;43:327-34.

28 Ramjee G, Morar NS, Braunstein S, et al. Acceptability of Carraguard, a candidate microbicide and methyl cellulose placebo 
vaginal gels among HIV-positive women and men in Durban, South Africa. AIDS Res Ther 2007;4:20.

29 van de Wijgert JHHM, Braunstein SL, Morar NS, et al. Carraguard vaginal gel safety in HIV-positive women and men in South Africa. $J$ Acquir Immune Defic Syndr 2007;46:538-46.

30 Dupont WD, Plummer WD. Power and sample size calculations. A review and computer program. Control Clin Trials 1990;11:116-28.

31 Macaluso M, Lawson ML, Hortin G, et al. Efficacy of the female condom as a barrier to semen during intercourse. Am J Epidemiol 2003;157:289-97.

32 Baer A, Saroiu S, Koutsky LA. Obtaining sensitive data through the web: an example of design and methods. Epidemiology 2002;13:640-5.

33 US Department of Health and Human Services NloH, National Institute of Allergy and Infectious Diseases D of A. Division of AIDS Table for Grading the Severity of Adult and Pediatric Adverse Events, Addendum 3 - Rectal Grading Table for Use in Microbicide Studies, Version 1.0, 2012

34 U.S. Department of Health and Human Services NloH, National Institute of Allergy and Infectious Diseases D of A. Division of AIDS Table for Grading the Severity of Adult and Pediatric Adverse Events, Addendum 2 - Male Genital Grading Table for Use in Microbicide Studies, Version 1.0, 2017.

35 Tarkowski TA, Rajeevan MS, Lee DR, et al. Improved detection of viral RNA isolated from liquid-based cytology samples. Mol Diagn 2001;6:125-30.

36 Coutlée F, Rouleau D, Petignat P, et al. Enhanced detection and typing of human papillomavirus (HPV) DNA in anogenital samples with PGMY primers and the linear array HPV genotyping test. $J$ Clin Microbiol 2006;44:1998-2006.

37 Shaw E, Ramanakumar AV, El-Zein M, et al. Reproductive and genital health and risk of cervical human papillomavirus infection: results from the Ludwig-McGill cohort study. BMC Infect Dis 2016;16:116.

38 Schiffman M, Clifford G, Buonaguro FM. Classification of weakly carcinogenic human papillomavirus types: addressing the limits of epidemiology at the borderline. Infect Agent Cancer 2009;4:8

39 Schiffman M, Herrero R, Desalle R, et al. The carcinogenicity of human papillomavirus types reflects viral evolution. Virology 2005;337:76-84.

40 Castle PE, Jeronimo J, Schiffman M, et al. Age-Related changes of the cervix influence human papillomavirus type distribution. Cancer Res 2006;66:1218-24.

41 Hernán MA. The hazards of hazard ratios. Epidemiology 2010;21:13-15

42 Magnan S, Tota JE, El-Zein M, et al. Efficacy of a carrageenan gel against transmission of cervical HPV (catch): interim analysis of a randomized, double-blind, placebo-controlled, phase 2B trial. Clin Microbiol Infect 2019;25:210-6.

43 Schulz KF, Altman DG, Moher D, et al. Consort 2010 statement: updated guidelines for reporting parallel group randomised trials. BMJ 2010;340:c332.

44 Gohy L, Gorska I, Rouleau D, et al. Genotyping of human papillomavirus DNA in anal biopsies and anal swabs collected from HIV-seropositive men with anal dysplasia. J Acquir Immune Defic Syndr 2008;49:32-9.

45 Hildesheim A, Herrero R, Wacholder S, et al. Effect of human papillomavirus 16/18 L1 viruslike particle vaccine among young women with preexisting infection: a randomized trial. JAMA 2007;298:743.

46 Mooij SH, van Santen DK, Geskus RB, et al. The effect of HIV infection on anal and penile human papillomavirus incidence and clearance: a cohort study among MSM. AIDS 2016;30:121-32.

47 Donà MG, Vescio MF, Latini $A$, et al. Anal human papillomavirus in HIV-uninfected men who have sex with men: incidence and clearance rates, duration of infection, and risk factors. Clin Microbiol Infect 2016;22:1004.e1-1004.e7.

48 Marra E, Kovaleva A, Bruisten SM, et al. Incidence and clearance of anal high-risk human papillomavirus infections and their determinants over 5 years among human immunodeficiency virus-negative men who have sex with men. Clin Infect Dis 2019;68:1556-65.

49 Darwich L, Cañadas M-P, Videla S, et al. Prevalence, clearance, and incidence of human papillomavirus type-specific infection at the anal and penile site of HIV-infected men. Sex Transm Dis 2013;40:611-8.

50 Videla S, Darwich L, Cañadas M-P, et al. Natural history of human papillomavirus infections involving anal, penile, and oral sites among HIV-positive men. Sex Transm Dis 2013;40:3-10.

51 Geskus RB, González C, Torres M, et al. Incidence and clearance of anal high-risk human papillomavirus in HIV-positive men who have sex with men: estimates and risk factors. AIDS 2016;30:37-44.

52 Patel P, Bush T, Conley L, et al. Prevalence, incidence, and clearance of human papillomavirus types covered by current vaccines in men with human immunodeficiency virus in the sun study. J Infect Dis 2019. doi:10.1093/infdis/jiz425. [Epub ahead of print: 19 Sep 2019].

53 Alberts CJ, Heard I, Canestri A, et al. Incidence and clearance of ana HPV-16 and HPV-18 infection, and their determinants, among HIVinfected men who have sex with men in France. J Infect Dis 2019. doi:10.1093/infdis/iiz623. [Epub ahead of print: 22 Nov 2019].

54 Twisk DE, van der Sande MAB, van Eeden A, et al. Detection of incident anal high-risk human papillomavirus DNA in men who have sex with men: incidence or reactivation? J Infect Dis 2018;218:1018-26. 